\title{
Constrained Codes for Joint Energy and Information Transfer
}

\author{
Ali Mohammad Fouladgar, Student Member, IEEE, Osvaldo Simeone, Member, IEEE, and Elza Erkip, Fellow, IEEE
}

\begin{abstract}
In various wireless systems, such as sensor RFID networks and body area networks with implantable devices, the transmitted signals are simultaneously used both for information transmission and for energy transfer. To satisfy the conflicting requirements on information and energy transfer, this paper proposes the use of constrained run-length limited (RLL) codes in lieu of conventional unconstrained (i.e., random-like) capacityachieving codes. The receiver's energy utilization requirements are modeled stochastically, and constraints are imposed on the probabilities of battery underflow and overflow at the receiver. It is demonstrated that the codewords' structure afforded by the use of constrained codes enables the transmission strategy to be better adjusted to the receiver's energy utilization pattern, as compared to classical unstructured codes. As a result, constrained codes allow a wider range of trade-offs between the rate of information transmission and the performance of energy transfer to be achieved.
\end{abstract}

Index Terms-Wireless energy transfer, constrained codes, run-length limited codes, renewal processes.

\section{INTRODUCTION}

$\mathbf{V}$ ARIOUS modern wireless systems, such as sensor RFID networks [1] and body area networks with implantable devices [2]-[4], challenge the conventional assumption that the energy received from an information bearing signal cannot be reused. For instance, implantable devices can be powered by the received radio signal, hence alleviating the need for a battery and reducing significantly the size of the devices. This realization has motivated a number of research groups to investigate the design of wireless systems under joint information and energy transfer requirements. ${ }^{1}$

The research activity in this area has focused so far on optimal resource allocation in the presence of information and energy transfer for various network topologies. Specifically, [8] studied a single point-to-point channel, while [9], [10] investigated power allocation for a set of parallel point-to-point chan-

Manuscript received November 6, 2013; revised February 11, 2014; accepted April 7, 2014. Date of publication April 15, 2014; date of current version June 18, 2014. The associate editor coordinating the review of this paper and approving it for publication was B. Clerckx.

A. M. Fouladgar is with the Center for Wireless Communications and Signal Processing Research (CWCSPR), New Jersey Institute of Technology, Newark, NJ07102 USA (e-mail: af82@njit.edu).

O. Simeone is with the New Jersey Institute of Technology, Newark, NJ 07103 USA (e-mail: osvaldo.simeone@njit.edu).

E. Erkip is with New York University Polytechnic School of Engineering, Brooklyn, NY 11201 USA (e-mail: elza@poly.edu).

Digital Object Identifier 10.1109/TCOMM.2014.2317480

${ }^{1}$ It is worth noting that wireless energy transfer, has a long history [5] and is available commercially (see, e.g., [6], [7]).

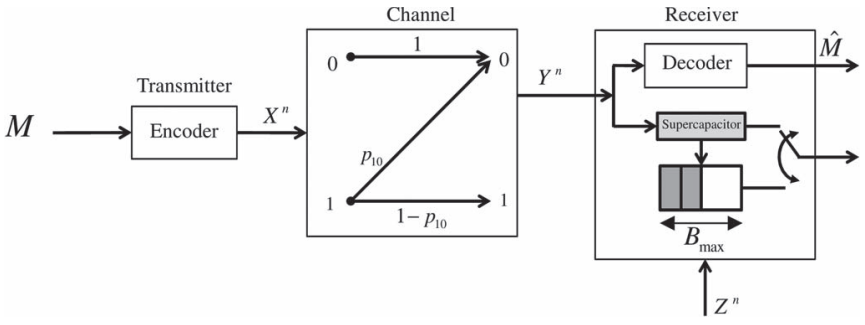

Fig. 1. Point-to-point link with information and energy transfer

nels under energy transfer and information rate constraints. The optimization of beamforming strategies under the same criteria was studied in [11]-[14] for multiantenna broadcast channels and for two-user multiantenna interference channels in [15]. Optimal resource allocation assuming wireless energy transfer was also investigated in [16] for cellular systems, in [17]-[19] for relay systems, in [20] for two-way interactive channels, and in [21] for graphical multi-hop networks. Considerations on the design of the receiver under the constraint that, when harvesting energy from the antenna, the receiver is not able to use the same signal for information decoding, can be found in [22].

Unlike all prior work summarized above, this work focuses on the code design for systems with joint information and energy transfer. We focus on a point-to-point link as shown in Fig. 1, in which the receiver's energy requirements are modeled as a random process. The statistics of this process generally depend on the specific application to be run at the receiver, e.g., sensing or radio transmission. The performance in terms of energy transfer is measured by the probabilities of overflow and underflow of the battery at the receiver. The probability of overflow measures the efficiency of energy transfer by accounting for the energy wasted at the receiver. Instead, the probability of underflow is a measure of the fraction of the time in which the application run at the receiver is in outage due to the lack of energy.

Classical codes, which are designed with the only aim of maximizing the information rate, are unstructured (i.e., random-like), see, e.g., [23]. As a result, they do not allow to control the timing of the energy transfer, and hence to optimize the probability of overflow and underflow. With this in mind, here it is proposed to adopt constrained run-length limited (RLL) codes [24] in lieu of conventional unconstrained codes. The constraints defining RLL codes ensure that the code does not includes bursts of energy either too frequently, thus limiting battery overflow, or too infrequently, thus controlling battery underflow. Constrained RLL codes have been traditionally studied for applications related to magnetic and optical storage [24]. The application to the problem at hand of energy 
transfer has been previously studied in the context of pointto-point RFID systems in [25], although no analysis of the information-energy trade-off was provided. In contrast, in this work, a thorough analysis is provided of the interplay between information rate and energy transfer in terms of probabilities of battery overflow and underflow. The analysis reveals that, by properly choosing the parameters that define RLL codes depending on the receiver's utilization requirements, constrained codes allow to greatly improve the system performance in terms of simultaneous energy and information transfer.

The remainder of this paper is organized as follows. In Section II, the system model is introduced along with performance criteria. In Sections III and IV, we study the performance of classical unconstrained codes and of constrained RLL codes, respectively, in terms of energy and information transfer. Section V presents numerical results. Finally, some concluding remarks can be found in Section VI.

\section{SYSTEM MODEL}

We consider the point-to-point channel illustrated in Fig. 1. We assume that at each discrete time $i$, the transmitter can either send an "on" symbol $X_{i}=1$, which costs one unit of energy, or an "off" signal $X_{i}=0$, which does not require any energy expenditure. The receiver either obtains an energycarrying signal, which is denoted as $Y_{i}=1$, or receives no useful energy, which is represented as $Y_{i}=0$. The channel is memoryless, and has transition probabilities as shown in Fig. 1. Accordingly, $p_{10}$ represents the probability that energy is lost when propagating between transmitter and receiver. ${ }^{2}$ At the receiver side, upon reception of an energy-carrying signal $Y_{i}=1$, the energy contained in the signal is harvested. The harvested energy is temporarily held in a supercapacitor and, if not used in the current time interval $i$, is stored in a battery, whose capacity limited to $B_{\max }$ energy units (see, e.g., [26]).

The receiver's energy utilization is modeled as a stochastic process $Z_{i} \in\{0,1\}$, so that $Z_{i}=1$ indicates that the receiver requires one unit of energy at time $i$, while $Z_{i}=0$ implies that no energy is required by the receiver at time $i$. This process is not known at the transmitter and evolves according to the Markov chain shown in Fig. 2. Note that adopting a Markov model to account for the time variations of energy usage is a standard practice (see, e.g., [27] and references therein). Accordingly, when in state $U_{0}$, there may be bursts of consecutive time instants in which no energy is required (i.e., $Z_{i}=0$ ); while, when in state $U_{1}$, there may be bursts of consecutive time instants in which energy is required (i.e., $Z_{i}=1$ ). The probability that $Z_{i}=0$ when in state $U_{0}$ is referred to as $q_{0}$ and the probability that $Z_{i}=1$ in state $U_{1}$ is denoted as $q_{1}$. We observe that the average length of bursts of symbols in which $Z_{i}=j$ in state $U_{j}$ is given by $1 /\left(1-q_{j}\right)$ for $j \in\{0,1\}$. Also, it is remarked that, when $q_{0}=1-q_{1}$, the energy usage model becomes a memoryless process with $\operatorname{Pr}\left[Z_{i}=1\right]=1-q_{0}=q_{1}$.

\footnotetext{
${ }^{2}$ A more general model would allow also for a non-zero probability $p_{01}$ of receiving energy when no energy is transmitted. This could be interpreted as the probability of harvesting energy from the environment (see [20]). We do not consider this extension in this work.
}

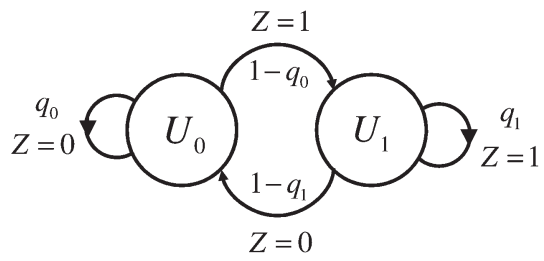

Fig. 2. Energy utilization model at the receiver.

Due to the finite capacity of the battery, there may be battery overflows and underflows. An overflow event takes place when energy is received and stored in the supercapacitor (i.e., $Y_{i}=$ 1 ), but is not used by the receiver (i.e., $Z_{i}=0$ ) and the battery is full (i.e., $B_{i}=B_{\max }$ ), so that the energy unit is lost; instead, an underflow event occurs when energy is required by the receiver (i.e., $Z_{i}=1$ ) but the supercapacitor and the battery are empty (i.e., $B_{i}=0$ and $Y_{i}=0$ ). In the rest of this section we define all the parts of the system in Fig. 1 in detail.

\section{A. Transmitter}

The transmitter aims at communicating a message $M$, uniformly distributed in the set $\left[1: 2^{n R}\right]$, reliably to the decoder, while at the same time guaranteeing desired probabilities of battery overflow and underflow (see Section II-B). Note that $n$ is the codeword length and $R$ represents the information rate in bits per channel use, while the constraints on the probabilities of overflow and underflow represents the requirements on energy transfer. As discussed in Section I, in this work, we investigate the performance in terms of information and energy transfer achievable of conventional unconstrained codes and of RLL codes. We introduce RLL codes next following [24].

The codewords $x^{n}(m)$, with $m \in\left[1: 2^{n R}\right]$, of a type- $i$ RLL code satisfy run-length constraints on the number of consecutive symbols $i$, where $i=0$ or $i=1 .^{3}$ To elaborate, let $d$ and $k$ be integers such that $0 \leq d \leq k$. We say that a finite length binary sequence $x^{n}(m)$ satisfies the type-0 $(d, k)$-RLL constraint if the following two conditions hold (see Fig. 3):

- the runs of 0's have length at most $k$, and

- the runs of 0 's between successive 1's have length at least $d$; note that the first and last runs of 0 's are allowed to have lengths smaller than $d$.

Therefore, a type- $0(d, k)$-RLL code is such that the codewords include sufficiently long stretches of zero-energy symbols 0 , via the selection of $d$, thus limiting battery overflow, but not too infrequently, via $k$, thus partly controlling also battery underflow. As a result, type- $0(d, k)$-RLL codes are suitable for overflow-limited regimes in which controlling overflow events is most critical. An example of a sequence satisfying the type- $0(d, k)=(2,7)$-RLL constraint is $x^{n}(m)=$ 00100001001000000010 where $n=20$. Overall, the set of all sequences $x^{n}(m)$ satisfying a type- $0(d, k)$-RLL constraint is then described by all the possible $n$-bit outputs of the finite state machine in Fig. 3, where the outputs are shown by the binary

\footnotetext{
${ }^{3}$ Classical RLL codes as discussed in, e.g., [24] are type-0, but here we find it useful to extend the definition to include also type-1 RLL codes.
} 


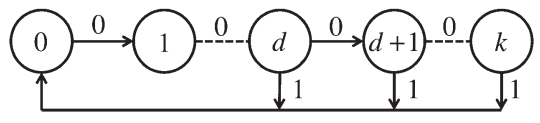

Fig. 3. Codewords of a type- $0(d, k)$-RLL code must be outputs of the shown finite-state machine. A type-1 $(d, k)$-RLL constrained code is instead obtained by substituting all " 0 " for " 1 " and vice versa.

labels of the directed edges. Note that finite-state machine consists of $k+1$ states (the numbered circles) and the initial state is arbitrary.

A type-1 $(d, k)$-RLL code is defined in the same way, upon substitution of all " 0 " for " 1 " and vice versa in the edge labels of Fig. 3. Therefore, type-1 $(d, k)$-RLL codes allow one to control the stretches of "1" symbols in the codewords, and are hence well suited for underflow-limited regimes, in which controlling the probability of underflow is most important.

\section{B. Receiver}

Transmitter and receiver communicate over the binary channel shown in Fig. 1 with the probability of $p_{10}$ of flipping symbol " 1 " to symbol " 0 ". As mentioned, this probability can be interpreted in terms of energy losses across the channel. The received signal $Y^{n}$ is used by the decoder both to decode the information message $M$ encoded via the constrained code at the transmitter and to perform energy harvesting. The harvested energy is used to fulfill the energy requirements of the receiver as dictated by the process $Z^{n}$, where the requirements are given in terms of probability of overflow and underflow. This is discussed next.

Let $B_{i}$ denote the number of energy units available in the battery at time $i$. At the $i$ th time period, the decoder first receives signal $Y_{i}$, and stores its energy (if $Y_{i}=1$ ) temporarily in a supercapacitor (see Fig. 1). Then, if $Z_{i}=1$, the receiver attempts to draw one energy unit from the supercapacitor or, if the latter is empty, from the battery. If the energy in the supercapacitor is not used, it is stored in the battery in the next time slot. As a result, the amount of energy in the battery evolves as

$$
B_{i+1}=\min \left(B_{\max },\left(B_{i}+Y_{i}-Z_{i}\right)^{+}\right),
$$

where $(a)^{+}=\max (0, a)$.

When the receiver harvests a unit of energy, $Y_{i}=1$, no energy is used, $Z_{i}=0$, and the battery is full, $B_{i}=B_{\max }$, we have an overflow event. To keep track of the overflow events, we define a random process $O_{i}$ such that $O_{i}=1$ if the event $\left\{B_{i}=B_{\max }, Y_{i}=1\right.$, and $\left.Z_{i}=0\right\}$ occurs and $O_{i}=0$ otherwise. This can be expressed as

$$
O_{i}=1\left\{B_{i}=B_{\max }, Y_{i}=1 \text { and } Z_{i}=0\right\} .
$$

When the receiver wishes to use a unit of energy, $Z_{i}=1$, and both the supercapacitor and the battery are empty, $Y_{i}=0$ and $B_{i}=0$, we have an underflow event. To describe underflow events, we introduce a random process $U_{i}$ such that $U_{i}=1$ if

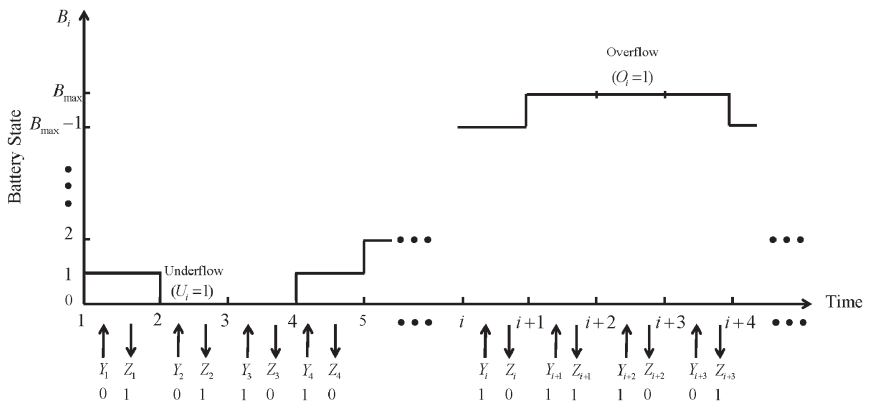

Fig. 4. Sample path of the evolution of the battery $B_{i}$. Also, indicated is the assumed order of energy arrival and departure events from the battery (i.e., $Y_{i}$ and $Z_{i}$ ) and the overflow and underflow events (where not specified, we have $U_{i}=0$ and $\left.O_{i}=0\right)$.

the event $\left\{B_{i}=0, Y_{i}=0\right.$ and $\left.Z_{i}=1\right\}$ takes place and $U_{i}=0$ otherwise. This can be expressed as

$$
U_{i}=1\left\{B_{i}=0, Y_{i}=0 \text { and } Z_{i}=1\right\} .
$$

A sample path of the battery state process $B_{i}$ along with $Y_{i}, Z_{i}$, $U_{i}$ and $O_{i}$, is shown in Fig. 4 .

We define the probability of underflow as

$$
\operatorname{Pr}\{\mathcal{U}\}=\limsup _{n \rightarrow \infty} \frac{1}{n} \sum_{i=1}^{n} \mathrm{E}\left[U_{i}\right]
$$

and the probability of overflow as

$$
\operatorname{Pr}\{\mathcal{O}\}=\limsup _{n \rightarrow \infty} \frac{1}{n} \sum_{i=1}^{n} \mathrm{E}\left[O_{i}\right] .
$$

We note that in (4) and (5), the expectation is taken over the distribution of the message $M$, of the channel and of the receiver's energy utilization process $Z^{n}$.

\section{Performance Criteria and Problem Formulation}

The point-to-point link under study will be evaluated in terms of its performance for both information and energy transfer. A triple $\left(R, P_{o f}, P_{u f}\right)$ of information-energy requirements is said to be achievable by an encoder-decoder pair if the information transfer at rate $R$ is reliable, i.e., if

$$
\limsup _{n \rightarrow \infty} \operatorname{Pr}[\hat{M} \neq M]=0
$$

and if the energy transfer fulfill the constraints

$$
\begin{aligned}
& \operatorname{Pr}\{\mathcal{O}\} \leq P_{o f}, \\
& \operatorname{Pr}\{\mathcal{U}\} \leq P_{u f} .
\end{aligned}
$$

We are interested in investigating the set of achievable triples $\left(R, P_{o f}, P_{u f}\right)$ for different classes of codes, namely unconstrained and $(d, k)$-RLL constrained. To obtain further insight, in Section V, we will consider the problem

$$
\begin{array}{ll}
\operatorname{minimize} & \max \left(P_{o f}, P_{u f}\right) \\
\text { subject to } & \left(R, P_{o f}, P_{u f}\right) \quad \text { is achievable, }
\end{array}
$$




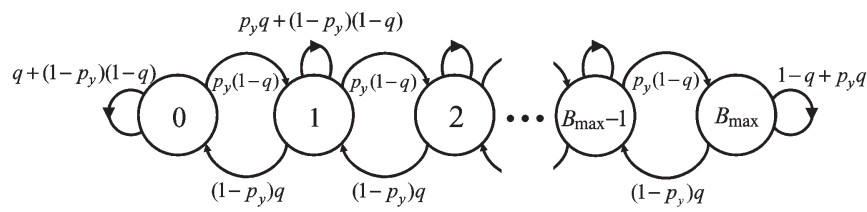

Fig. 5. Birth-death Markov process defining the battery state evolution along the channel uses with unconstrained (i.i.d.) random codes and i.i.d. receiver's energy usage process $Z^{n}$ (i.e., $q=q_{1}=1-q_{0}$ ).

where $R$ is fixed and the minimization is done over all codes belonging to a certain class. Problem (9) is appropriate when both underflow and overflow are equally undesirable and one wishes to reduce both equally as much as possible. Alternatively, one could, e.g., minimize either one of $P_{o f}$ or $P_{u f}$ under a given constraint on the other and on the rate.

\section{UNCONSTRAINED CODES}

In this section, we study the information-energy transfer performance of classical unconstrained codes. To this end, we adopt Shannon's classical random coding argument. Accordingly, we assume that the codewords $x^{n}(m), m \in\left[1: 2^{n R}\right]$, are generated independently as i.i.d. $\operatorname{Ber}\left(p_{x}\right)$ processes and evaluate the corresponding performance on average over the code ensemble. As it is well known (see, e.g., [23]), the maximum information rate $R$ achieved by this code is given as

$$
\begin{aligned}
R & =\mathrm{I}(X ; Y) \\
& =\mathrm{H}(Y)-\mathrm{H}(Y \mid X) \\
& =\mathrm{H}\left(p_{x}\left(1-p_{10}\right)\right)-p_{x} \mathrm{H}\left(p_{10}\right) \\
& =\mathrm{H}\left(p_{y}\right)-\frac{p_{y}}{1-p_{10}} \mathrm{H}\left(p_{10}\right),
\end{aligned}
$$

where we have defined the probability $p_{y} \triangleq \operatorname{Pr}\left[Y_{i}=1\right]=$ $p_{x}\left(1-p_{10}\right)$ and the binary entropy function

$$
\mathrm{H}(a) \triangleq-a \log _{2} a-(1-a) \log _{2}(1-a) .
$$

We now turn to the evolution of the performance in terms of energy transfer. To simplify the analysis and obtain some insight, we first assume the special case for the receiver's energy utilization model in which the process $Z^{n}$ is i.i.d. and hence $q_{1}=1-q_{0} \triangleq q$. Note that $q$ is the energy usage probability, in that we have $q=\operatorname{Pr}\left[Z_{i}=1\right]$. The extension to the more general Markov model of Fig. 2 will be discussed in Remark 3. If the process $Z^{n}$ is i.i.d., the battery state evolves according to the birth-death Markov chain shown in Fig. 5. Recalling (2) and (3), we can then calculate the probability of overflow and underflow, respectively, as

$$
\begin{aligned}
& \operatorname{Pr}\{\mathcal{O}\}=\pi_{B_{\max }} p_{y}(1-q) \triangleq \mathrm{O}\left(p_{y}\right), \\
& \operatorname{Pr}\{\mathcal{U}\}=\pi_{0}\left(1-p_{y}\right) q \triangleq \mathrm{U}\left(p_{y}\right),
\end{aligned}
$$

where $\pi_{i}$ is the steady-state probability of state $i \in\left[0, B_{\max }\right]$ for the Markov chain in Fig. 5. Note that (12) follows from the independence of the events $\left\{B_{i}=B_{\max }\right\},\left\{Y_{i}=1\right\}$ and $\left\{Z_{i}=0\right\}$ and similarly for (13). The probability $\pi_{i}$ can be calculated as (see, e.g., [28, Ch. 4])

$$
\pi_{i}=\frac{A^{i}}{1+A+\cdots+A^{B_{\max }}},
$$

where $A=p_{y}(1-q) /\left(1-p_{y}\right) q$. By combining the equations (10), (12), and (13), we obtain the following lemma.

Lemma 1: Given a receiver energy usage i.i.d. process with energy usage probability $q$, the set of achievable informationenergy triples $\left(R, P_{o f}, P_{u f}\right)$ for unconstrained (i.i.d.) codes is given by

$$
\begin{aligned}
& \left\{\left(R, P_{o f}, P_{u f}\right): \exists p_{y} \in\left[0,1-p_{10}\right]\right. \text { such that } \\
& R \leq \mathrm{H}\left(p_{y}\right)-\frac{p_{y}}{1-p_{10}} \mathrm{H}\left(p_{10}\right) \\
& \left.P_{o f} \geq \mathrm{O}\left(p_{y}\right), P_{u f} \geq \mathrm{U}\left(p_{y}\right)\right\},
\end{aligned}
$$

where $\mathrm{H}\left(p_{y}\right), \mathrm{O}\left(p_{y}\right)$ and $\mathrm{U}\left(p_{y}\right)$ are defined in (11)-(13), respectively.

Remark 1: The region (15) is in general not convex, but it can be convexified if one allows for time sharing between codes with different values of $p_{y}$ (see, e.g., [29, Ch. 4] for related discussion).

To get further insight into the performance of unconstrained codes, we now assume that the channel is noiseless, i.e., $p_{10}=0$ and, as a result, we have $Y_{i}=X_{i}$ for all $i=1, \ldots, n$ and $p_{y}=p_{x}$. Moreover, we consider problem (9), which reduces to the following optimization problem:

$$
\begin{aligned}
& \underset{p_{x} \in[0,1]}{\operatorname{minimize}} \max \left(\mathrm{O}\left(p_{x}\right), \mathrm{U}\left(p_{x}\right)\right) \\
& \text { subject to : } \mathrm{H}\left(p_{x}\right) \geq R
\end{aligned}
$$

where $R$ is fixed. The solution of problem (17) is summarized in the following lemma.

Lemma 2: The optimal solution $p_{x}^{\star}$ of problem (17) is given as

$$
\begin{aligned}
q & \text { if } R \leq \mathrm{H}(q) \\
\mathrm{H}^{-1}(R) & \text { if } R>\mathrm{H}(q) \text { and } q \leq \frac{1}{2} \\
1-\mathrm{H}^{-1}(R) & \text { if } R>\mathrm{H}(q) \text { and } q>\frac{1}{2}
\end{aligned}
$$

where $\mathrm{H}^{-1}(R)$ is the inverse of the entropy function in the interval $[0,1 / 2]$. Moreover, the optimal value $\max \left(\mathrm{O}\left(p_{x}^{\star}\right), \mathrm{U}\left(p_{x}^{\star}\right)\right)$ of the problem (17) is given by

$$
\begin{array}{cl}
\frac{(1-q) q}{B_{\max }+1} & \text { if } R \leq \mathrm{H}(q) \\
\mathrm{O}\left(\mathrm{H}^{-1}(R)\right) & \text { if } R>\mathrm{H}(q) \text { and } q \leq \frac{1}{2} \\
\mathrm{U}\left(1-\mathrm{H}^{-1}(R)\right) & \text { if } R>\mathrm{H}(q) \text { and } q>\frac{1}{2} .
\end{array}
$$




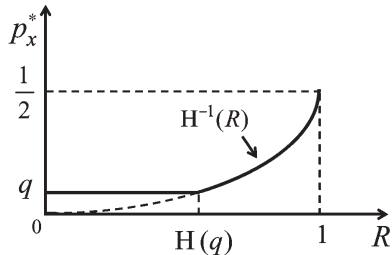

(a)

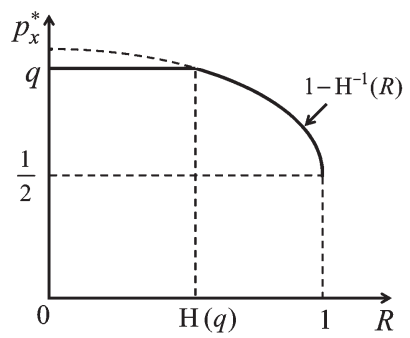

(b)

Fig. 6. Illustration of the optimal solution $p_{x}^{\star}$ of problem (17) (Lemma 2): (a) the overflow-limited regime $q \leq 1 / 2$; (b) the underflow-limited regime $q>1 / 2$.

Proof: A graphical illustration of Lemma 2 is shown in Fig. 6. To interpret the conditions (18a) and (19a), we observe that the underflow probability $\mathrm{U}\left(p_{x}\right)$ is monotonically decreasing with $p_{x}$, while the overflow probability $\mathrm{O}\left(p_{x}\right)$ is monotonically increasing with $p_{x}$. Therefore, in the absence of the rate constraint, the optimal value of problem (17) is achieved when $p_{x}=q$, since, with this choice, we have $\mathrm{O}\left(p_{x}\right)=\mathrm{U}\left(p_{x}\right)$. As a result, if $R \leq \mathrm{H}(q)$, and hence the rate constraint is immaterial for $p_{x}=q$, we have $p_{x}^{\star}=q$.

Instead, if $R>\mathrm{H}(q)$, the rate constraint is active and the optimal solution requires $R=\mathrm{H}\left(p_{x}\right)$. In particular, there are two situations to be considered, namely the overflow-limited regime, defined by the condition $q \leq 1 / 2$, and the underflowlimited regime, where we have $q>1 / 2$. In the former regime (Fig. 6(a)), the rate constraint forces $p_{x}$ to be larger than $q$, which leads to the optimal solution $p_{x}^{\star}=\mathrm{H}^{-1}(R)$ and causes the overflow probability $\mathrm{O}\left(p_{x}\right)$ to be larger than the underflow probability $\mathrm{U}\left(p_{x}\right)$, so that $\max \left(\mathrm{O}\left(p_{x}\right), \mathrm{U}\left(p_{x}\right)\right)=\mathrm{O}\left(p_{x}\right)$. In contrast, in the underflow-limited regime (Fig. 6(b)), the rate constraint forces $p_{x}$ to be smaller than $q$, which leads to $p_{x}^{\star}=1-\mathrm{H}^{-1}(R)$ and causes $\mathrm{U}\left(p_{x}\right)$ to dominate $\mathrm{O}\left(p_{x}\right)$, or $\max \left(\mathrm{O}\left(p_{x}\right), \mathrm{U}\left(p_{x}\right)\right)=\mathrm{U}\left(p_{x}\right)$.

Remark 2: The proof of Lemma 2 suggests that, when the rate is sufficiently small, problem (9) is solved by "matching" the code structure to the receiver's energy utilization model. This is done, under the given i.i.d. assumption on codes and receiver's energy utilization, by setting $p_{x}^{\star}=q$. Instead, when the rate constraint is the limiting factor, one is forced to allow for a mismatch between code properties and receiver's energy utilization model (by setting $p_{x}^{\star} \neq q$ ). These ideas will be useful when interpreting the gains achievable by constrained codes discussed in Section IV.

Remark 3: The characterization of the achievable information-energy triples $\left(R, P_{o f}, P_{u f}\right)$ of Lemma 1 can be extended to the more general Markov model in Fig. 2 for the receiver's energy usage. This is done by noting that the battery evolution under this model is described by the Markov chain shown in Fig. 7, instead of the simpler

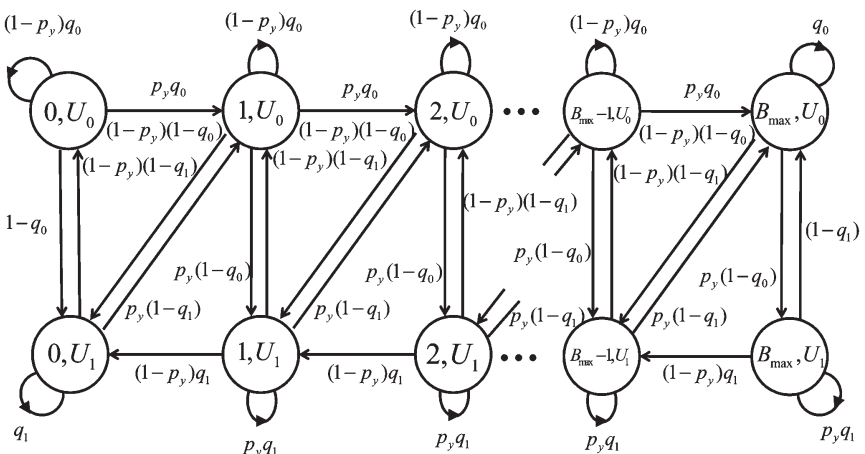

Fig. 7. Markov process defining the battery state evolution along the channel uses with unconstrained (i.i.d.) random codes and the Markov receiver's energy usage model of Fig. 2.

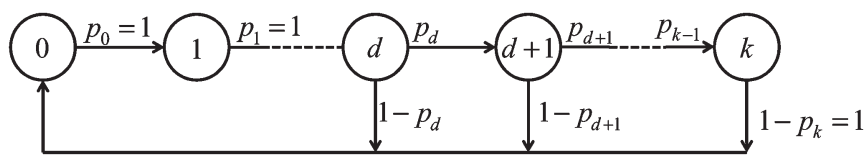

Fig. 8. Transition probabilities $\mathcal{P}=\left\{p_{d}, p_{d+1}, \ldots, p_{k-1}\right\}$ defining the stationary Markov chain used for random coding with type-0 $(d, k)$-RLL codes.

birth-death Markov process shown in Fig. 5. The calculation of the corresponding steady-state probabilities $\pi_{i, U_{j}}$, for $i \in\left[0, B_{\max }\right]$ and $j=\{0,1\}$, can be done using standard steps (see, e.g., [28]). Lemma 1 then extends to the scenario at hand by calculating the probabilities of overflow and underflow, similar to (12), (13) as

$$
\begin{aligned}
\operatorname{Pr}\{\mathcal{O}\}= & \pi_{B_{\max }, U_{0}} p_{y} q_{0} \\
& +\pi_{B_{\max }, U_{1}} p_{y}\left(1-q_{1}\right) \triangleq \mathrm{O}\left(p_{y}\right), \\
\operatorname{Pr}\{\mathcal{U}\}= & \pi_{0, U_{0}}\left(1-p_{y}\right)\left(1-q_{0}\right) \\
& +\pi_{0, U_{1}}\left(1-p_{y}\right) q_{1} \triangleq \mathrm{U}\left(p_{y}\right) .
\end{aligned}
$$

\section{Constrained Codes}

In this section, we study the performance of $(d, k)$-RLL codes. To this end, as with unconstrained codes, we adopt a random coding approach. Specifically, we take the codewords to be generated independently according to a stationary Markov chain defined on the finite state machine in Fig. 3. It is known that this choice is optimal in terms of capacity (see, e.g., [24], [25], [30]). A stationary Markov chain on the graph of Fig. 3 is defined by the transition probabilities $\mathcal{P}=\left\{p_{d}, p_{d+1}, \ldots, p_{k-1}\right\}$ on its edges as shown in Fig. 8 . We define as $C_{i}$ the state of the constrained code at time $i$, prior to the transmission of $X_{i}$. For example, the state sequence for the type- $0(d, k)=(2,7)$-RLL corresponding to the codeword $x^{n}(m)=00100001001000000010$ is $c^{n}(m)=$ 01201234012012345670 where $n=20$. Then, the transition probability $p_{j}$ for $j=d, \ldots, k-1$ is equal to $\operatorname{Pr}\left[C_{i}=j+\right.$ $1 \mid C_{i-1}=j$ ], for $i>1$. Barring degenerate choices for $\mathcal{P}$, it is easy to see that the Markov chain is irreducible, and hence one can calculate the unique steady-state distribution $\pi_{j}=\operatorname{Pr}\left[C_{i}=\right.$ $j]$ for $j \in[0, k]$ (see, e.g., [24]). 


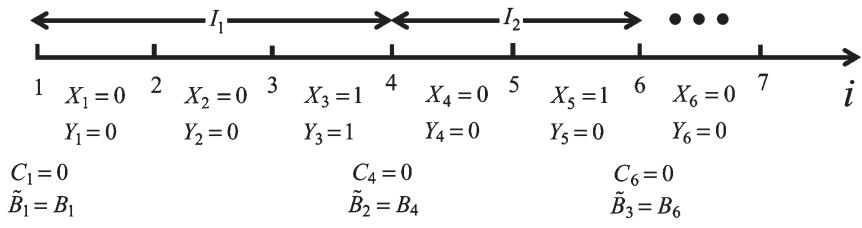

Fig. 9. Sample of renewal events $\left\{C_{i}=0\right\}$ and the corresponding evolution of the battery across channel uses for a type- $0(d, k)$-RLL code.

\section{A. Information Rate}

In [31, Lemma 5], it was proved that an achievable rate $R$ with $(d, k)$-RLL codes is given as $R=\mathrm{I}\left(C_{2} ; Y_{2} \mid C_{1}\right)$. Evaluating this expression for type- $0(d, k)$-RLL constrained codes leads to

$$
\begin{aligned}
R & =\mathrm{H}\left(Y_{2} \mid C_{1}\right)-\mathrm{H}\left(Y_{2} \mid C_{1}, C_{2}\right) \\
& =\sum_{j=d}^{k-1} \pi_{j}\left\{\mathrm{H}\left(\left(1-p_{j}\right)\left(1-p_{10}\right)\right)-\left(1-p_{j}\right) \mathrm{H}\left(p_{10}\right)\right\} .
\end{aligned}
$$

Instead, for type-1 code the achievable rate becomes

$$
R=\sum_{j=d}^{k-1} \pi_{j}\left\{\mathrm{H}\left(p_{j}\left(1-p_{10}\right)\right)-p_{j} \mathrm{H}\left(p_{10}\right)\right\}
$$

The maximization of the achievable information rate over the transition probabilities $\mathcal{P}$ with no regards for energy transfer is discussed in [24, Ch. 3].

\section{B. Energy Transfer}

We now address energy transfer by turning to the calculation of the probabilities of battery underflow and overflow, namely $\operatorname{Pr}\{\mathcal{U}\}$ and $\operatorname{Pr}\{\mathcal{O}\}$ in (4) and (5), respectively. To this end, as for unconstrained codes, we focus at first on the special case in which the energy usage process $Z^{n}$ is i.i.d. with energy usage probability $q$. We refer to Remark 4 below for a discussion on the extension to the Markov model in Fig. 2.

We use a renewal-reward argument (see, e.g., [28]). We recall that a renewal process is a random process of inter-renewal intervals $I_{1}, I_{2}, \ldots$ that are positive i.i.d. random variables. For our analysis, it is convenient to define the renewal event as $\left\{C_{i}=0\right\}$, so that a renewal takes place every time the state of the constrained code $C_{i}$ is equal to 0 . This is equivalent to saying that, in the channel use before a renewal event, the transmitted signal $X_{i}$ equals 1 for type-0 $(d, k)$-RLL codes and $X_{i}=0$ for type-1 $(d, k)$-RLL codes. We refer to Fig. 9 for an illustration. Based on the above, the renewal intervals $I_{i}$, for $i \geq 1$, are i.i.d. integer random variables with distribution $p_{I}(i)$ that can be calculated, given $\mathcal{P}$, as

$$
p_{I}(i)= \begin{cases}0 & i \leq d \text { and } i>k+1 \\ 1-p_{d} & i=d+1 \\ \left(1-p_{i-1}\right) \prod_{l=d}^{i-2} p_{l} & d+1<i \leq k \\ \prod_{l=d}^{k-1} p_{l} & i=k+1 .\end{cases}
$$

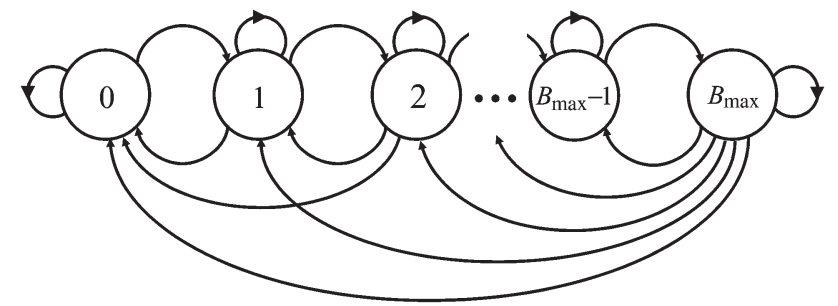

Fig. 10. Birth-death Markov process defining the battery state evolution along the renewal instants, where we have $C_{i}=0$, for $(d, k)$-RLL codes, i.i.d. receiver's energy usage pattern $Z^{n}$, and $k=B_{\max }$.

Moreover, it is useful to define a Markov chain $\tilde{B}_{i}$ that defines the evolution of the battery as evaluated at the renewal instants (i.e., for values of $i$ for which $C_{i}=0$ ), as illustrated in Fig. 9. We refer to the steady-state probability of this Markov chain as $\tilde{\pi}_{b}$ with $b \in\left[0, B_{\max }\right]$. Finally, we define as $\tilde{O}_{b}$ the random variable that counts the number of overflow events in a renewal that starts with a battery with capacity $b \in\left[0, B_{\max }\right]$, and, similarly, we define as $\tilde{U}_{b}$ the random variable that counts the number of underflow events in a renewal that starts with a battery with capacity $b \in\left[0, B_{\max }\right]$. We proceed by treating separately the type- 0 and type- 1 codes.

1) Type-0 Codes: For type-0 codes, the transition probabilities for the process $\tilde{B}_{i}$ are reported in Appendix A (see also Fig. 10 for an illustration), from which the steady state probabilities $\tilde{\pi}_{b}$ can be calculated (see, e.g., [28]). The next proposition summarizes the main result of the analysis. We use the definition $p(n ; i, q)=\left(\begin{array}{c}i \\ n\end{array}\right) q^{n}(1-q)^{i-n}$ with $n=0, \ldots, i$, for the probability distribution of a binomial random variable with parameters $(i, q)$.

Proposition 1: Given an i.i.d. receiver energy usage process with energy usage probability $q$, the set of achievable information-energy triples $\left(R, P_{o f}, P_{u f}\right)$ for type-0 $(d, k)$-RLL codes is given by

$$
\begin{aligned}
\left\{\left(R, P_{o f}, P_{u f}\right): \exists \mathcal{P}\right. \\
\qquad\left\{p_{d}, p_{d+1}, \ldots, p_{k-1}\right\} \in(0,1)^{n} \text { such that } R \\
\leq \sum_{j=d}^{k-1} \pi_{j}\left\{\mathrm{H}\left(\left(1-p_{j}\right)\left(1-p_{10}\right)\right)\right. \\
P_{o f} \geq \frac{\left.-\left(1-p_{j}\right) \mathrm{H}\left(p_{10}\right)\right\}}{\tilde{\pi}_{B_{\max }} \mathrm{E}\left[\tilde{O}_{B_{\max }}\right]} \\
\mathrm{E}[I] \\
\text { and } \left.P_{u f} \geq \frac{\sum_{\max } \tilde{\pi}_{b} \mathrm{E}\left[\tilde{U}_{b}\right]}{\mathrm{E}[I]}\right\}
\end{aligned}
$$


where we have defined

$$
\mathrm{E}[I]=\sum_{i=d+1}^{k+1} i \cdot p_{I}(i)
$$

along with

$$
\begin{aligned}
\mathrm{E}\left[\tilde{U}_{b}\right]= & \sum_{i=d+1}^{k+1} p_{I}(i) \\
& \times\left\{\left(1-p_{10}\right) \sum_{l=1}^{i-b-1} p(l+b ; i-1, q)\right. \\
& \left.+p_{10} \sum_{l=1}^{i-b} p(l+b ; i, q)\right\}, \\
\mathrm{E}\left[\tilde{O}_{\left.B_{\max }\right]}\right] & \sum_{i=d+1}^{k+1} p_{I}(i)\left(1-p_{10}\right) p(0 ; i, q) .
\end{aligned}
$$

Proof: See Appendix B.

Remark 4: The right-hand side of (27) evaluates the probability of overflow as the ratio of the average numbers of overflow events in a renewal interval over the average length of a renewal interval. The right-hand side of (28) can be similarly interpreted. Note that, by the given definition of renewal events, to have an overflow, the initial battery state $\tilde{B}_{i}$ must be in state $B_{\max }$, whereas underflow events can potentially happen for all states $b \in\left\{0, \ldots, B_{\max }\right\}$. This is reflected by the numerators of (27) and (28).

Similar to the case of unconstrained codes (see Remark 3), the characterization of the achievable information-energy triples $\left(R, P_{o f}, P_{u f}\right)$ of Proposition 1 can be extended to the more general Markov model in Fig. 2 for the receiver's energy usage. This is done by noting that the evolution of the battery state along the renewal instants can be still described by a Markov chain, albeit a more complex one. Moreover, to extend the analysis one needs to include in the state of the Markov process not only the battery state $\tilde{B}_{i}$ but also the state of the receiver's energy usage (either $U_{0}$ or $U_{1}$ ). The calculation of the corresponding transition probabilities is straightforward but cumbersome and is not detailed here.

2) Type-1 Codes: For type-1 codes, the analysis presented above does not easily generalize in the case in which the channel loss probability $p_{10}$ is nonzero. This can be seen by following the main steps of the proof of Proposition 1, which is based on having at most one non-zero received symbol per renewal interval. However, in the special case in which $p_{10}=0$, the approach can be generalized, leading to the following result.

Proposition 2: Given an i.i.d. receiver energy usage process with energy usage probability $q$, the set of achievable information-energy triples $\left(R, P_{o f}, P_{u f}\right)$ for type-1 $(d, k)$-RLL codes is given by

$$
\left\{\left(R, P_{o f}, P_{u f}\right): \exists \mathcal{P}=\left\{p_{d}, p_{d+1}, \ldots, p_{k-1}\right\} \in(0,1)^{n}\right.
$$

$$
\begin{array}{r}
R \leq \sum_{j=d}^{k-1} \pi_{j} \mathrm{H}\left(p_{j}\right), \\
P_{o f} \geq \frac{\sum_{b=0}^{B_{\max }} \tilde{\pi}_{b} \mathrm{E}\left[\tilde{O}_{b}\right]}{\mathrm{E}[I]}, \\
\text { and } \left.P_{u f} \geq \frac{\tilde{\pi}_{0} \mathrm{E}\left[\tilde{U}_{0}\right]}{\mathrm{E}[I]}\right\},
\end{array}
$$

where we have defined (29)

$$
\begin{aligned}
& \mathrm{E}\left[\tilde{U}_{0}\right]=\sum_{i=d+1}^{k+1} p_{I}(i) p(0 ; i, 1-q), \\
& \mathrm{E}\left[\tilde{O}_{b}\right]=\sum_{i=d+1}^{k+1} p_{I}(i) \sum_{l=1}^{i-b-1} p(l+b ; i-1,1-q) .
\end{aligned}
$$

Proof: Proposition 2 follows by the same steps as Proposition 1 and is not detailed here.

Remark 5: Similar to Proposition 1, the right-hand sides of (33), and of (34), evaluate the probabilities of overflow, and of underflow, via the ratios of the average numbers of overflow, and of underflow, events in a renewal interval over the average length of a renewal interval. In a dual fashion with respect to Proposition 1, given the definition of renewal events, underflow can only occur in renewal intervals with initial battery state $\tilde{B}_{i}$ is zero, whereas overflow events can potentially happen for all states $b \in\left\{0, \ldots, B_{\max }\right\}$.

\section{NumericAl Results}

In this section, we compare the performance of unconstrained and constrained codes using problem (9) as the benchmark. Fig. 11 shows the optimal value of $\max \left(P_{o f}, P_{u f}\right)$ for a noiseless channel, i.e., $p_{10}=0$ in Fig. 1 , when $R=0.1, q_{1}=0$ and $B_{\max }=2$ versus $q_{0}$ (recall Fig. 2). With $q_{1}=0$, the energy usage process $Z^{n}$ is such that a single energy request (i.e., $\left.Z_{i}=1\right)$ is followed by an average of $1 /\left(1-q_{0}\right)$ instants where no energy is required (i.e., $\left.Z_{i}=0\right)$. Therefore, as $q_{0}$ increases from 0.1 to 0.9 , the average length of an interval with no energy usage increases from around 1 to 10 . Similar to the discussion in Remark 2 for unconstrained codes, when neglecting the rate constraint, problem (9) is observed to be optimized by matching the code structure to the receiver's energy utilization model. When $q_{0}$ is sufficiently small, this can be easily accomplished with type- $0(d, k)$-RLL codes with a small $k$. This is because $k$ defines the maximum possible number of zero symbols $X_{i}$ sent before a symbol $X_{i}=1$. As $q_{0}$ increases, and hence the average length of the bursts of zeros grows in the process $Z_{i}$, the value of $k$ must be correspondingly increased. This is confirmed by Fig. 11, which shows the significant gain achievable by the use of RLL codes when properly selecting the code parameters. We observe that type-1 $(d, k)$-RLL codes would provide exactly the same performance in the symmetric case in which we have $q_{0}=0$, and hence intervals of energy usage (i.e., $Z_{i}=1$ ) are followed by a single instant with no energy usage (i.e., $Z_{i}=0$ ). 


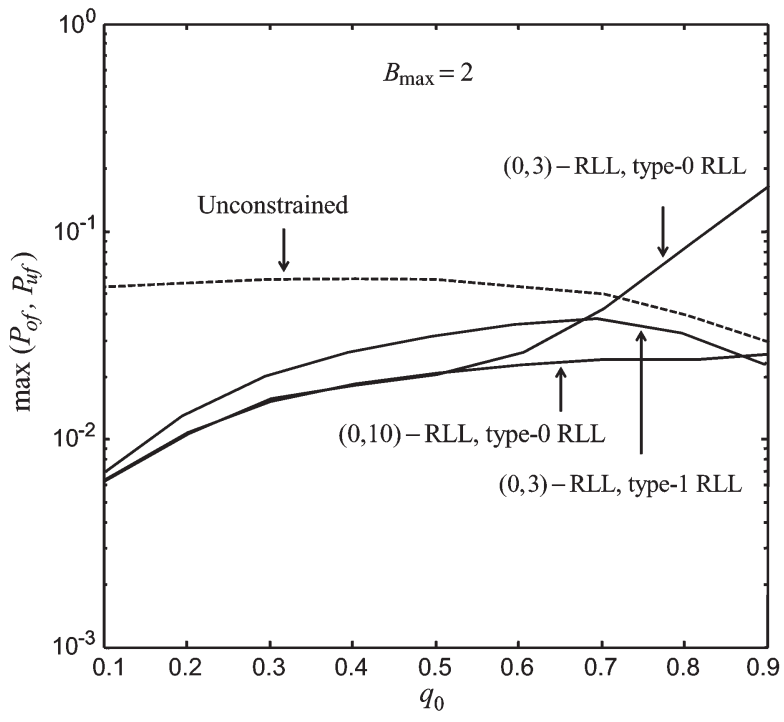

Fig. 11. Maximum between probability of underflow $P_{u f}$ and overflow $P_{o f}$ as per problem (9) for unconstrained and type- 0 constrained codes versus $q_{0}$ with $q_{1}=0$ (see Fig. 2) and $R=0.1$ and $B_{\max }=2$. To simplify the numerical optimization, the curve for $k=10$ has been obtained by optimizing only over $p_{0}, p_{1}, p_{2}, p_{3}$ and $p_{9}$ in $\mathcal{P}=\left\{p_{0}, p_{1}, \ldots, p_{9}\right\}$ and setting $p_{3}=$ $p_{4}=p_{5}=\cdots=p_{8}$.

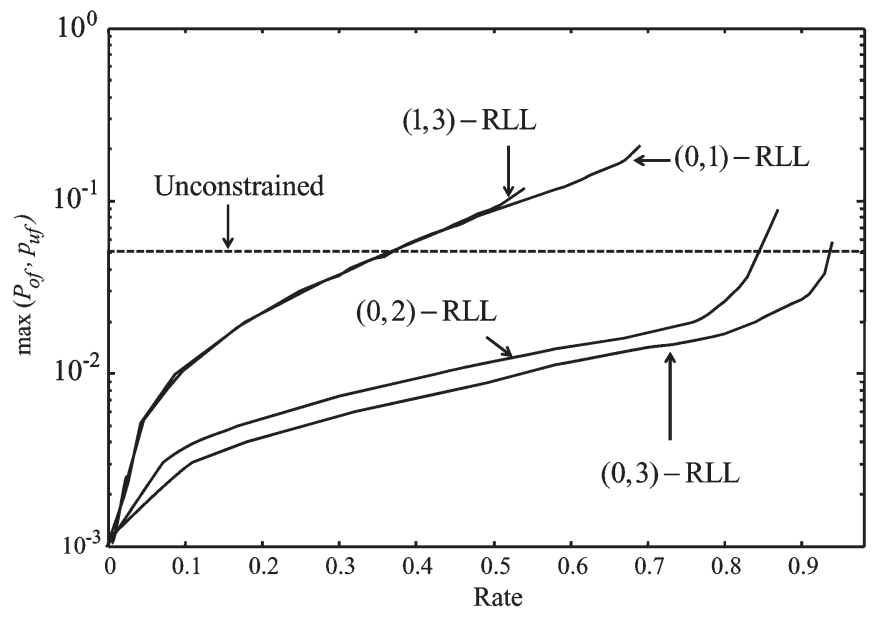

Fig. 12. Maximum between probability of underflow and overflow as per problem (9) for unconstrained and type- 0 constrained codes versus the information rate $R$ with $q_{0}=q_{1}=0$ (see Fig. 2), $p_{10}=0$ and $B_{\max }=2$.

Fig. 11 also shows the performance of type- 1 codes. It is seen that type- 1 codes are here advantageous.

The impact of the information rate $R$ is illustrated in Fig. 12 for $q_{0}=q_{1}=0, p_{10}=0$ and $B_{\max }=2$. Following the discussion above, when the rate is small, with $q_{0}=q_{1}=0$, it is sufficient to choose a type-0 or type- $1(d, k)$-RLL code with $k=1$, as this matches the energy usage process. However, as the rate grows larger, one needs to increase the value of $k$, while keeping $d$ as small as possible [24]. For instance, with $k=1$ and $d=0$, the maximum achievable rate is $R=0.6942$; with $k=2$ and $d=0$, it is $R=0.8791$; with $k=3$ and $d=1$, it is $R=0.5515$; and with $k=3$ and $d=0$, it is $R=0.9468$ [24, Table 3.1]. Accordingly, Fig. 12 shows again that, by appropriately choosing $d$ and $k$, RLL codes can provide relevant advantages.

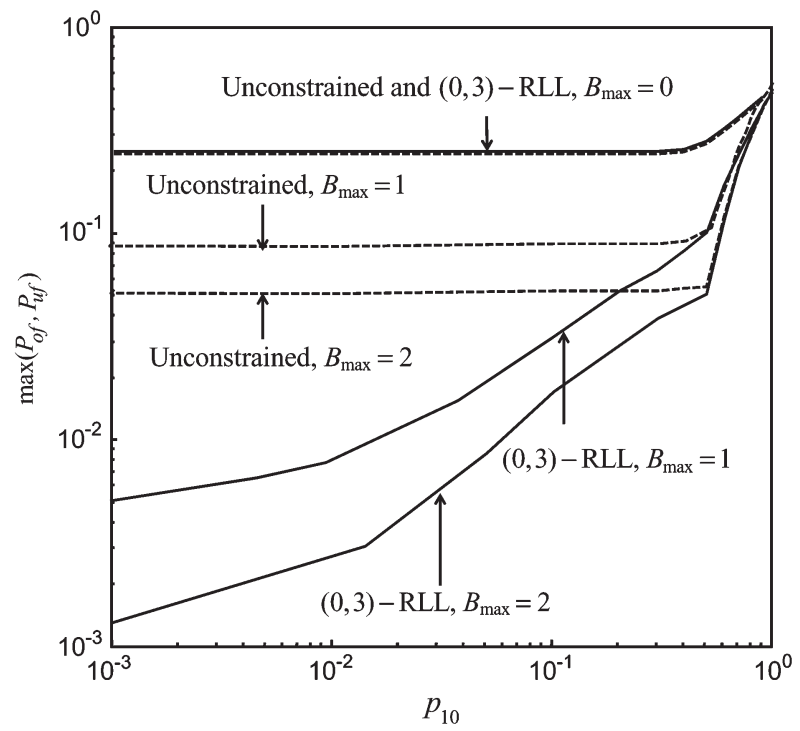

Fig. 13. Maximum between probability of underflow and overflow as per problem (9) for unconstrained and constrained codes versus $p_{10}$ for $k=3$, $R=0.01, q_{0}=q_{1}=0$ and $B_{\max }=0,1,2$.

Finally, we observe the effect of the loss probability $p_{10}$ in Fig. 13 where we set $R=0.01$ and $q_{0}=q_{1}=0$. Following the discussion above (see Remark 2), to match the receiver's energy utilization, the unconstrained code should be designed in such a way that $p_{y}=0.5$ since $\operatorname{Pr}\left[Z_{i}=1\right]=0.5$. Given that $p_{y}=p_{x}\left(1-p_{10}\right)$, this is only possible for $p_{10}<0.5$, and hence, for $p_{10}>0.5$, the performance is degraded as seen in Fig. 13. When $p_{10}=0$, as demonstrated above, RLL codes provide significant gains by providing a better matching to the utilization process $Z^{n}$. As the losses on the channel become more pronounced this gain decreases due to the reduced control of the received signal afforded by designing the transmitted signal. Fig. 13 also demonstrates the impact of the battery size $B_{\max }$, showing that the performance gains of constrained codes increase as the battery capacity grows from $B_{\max }=0$ to $B_{\max }=2$.

\section{CONCLUSION}

A host of new applications, including body area networks with implantable devices, is enabled by the possibility to reuse the energy received from information-bearing signals. With these applications in mind, we have investigated the use of constrained run-length limited (RLL) codes with the aim of enhancing the achievable performance in terms of simultaneous information and energy transfer. We have proposed a framework whereby the performance of energy transfer is measured by the probabilities of underflow and overflow at the receiver. The analysis has demonstrated that constrained codes enable the transmission strategy to be better adjusted to the receiver's energy utilization pattern as compared to classical unstructured codes. This has been shown to lead to significant performance gains especially at low information rates. Interesting future work includes the investigation of non-binary codes and multiterminal scenarios. 


\section{APPENDIX A}

TRANSITION PROBABILITIES FOR THE MARKOV CHAIN IN FIG. 10

Using the definitions in Section IV, we now calculate the transition probability $\tilde{p}_{m, m-n} \triangleq \operatorname{Pr}\left[\tilde{B}_{i}=m-n \mid \tilde{B}_{i-1}=m\right]$, where $\tilde{B}_{i}$ is the random process that describes the evolution of the battery at the renewal instants (see Fig. 9). The probabilities $\tilde{p}_{m, m-n}$ for $m \in\left[0, B_{\max }\right]$ and $n \in[-1, m]$ can be calculated as (37)-(40), shown at the bottom of the page.

\section{APPENDIX B}

\section{PROOF OF PROPOSITION 1}

In this Appendix, we prove Proposition 1 following a similar approach as [28, Theorem. 5.4.1]. We first relate the overflow event (2) and the underflow event (3) to the processes $\tilde{O}_{b}$ and $\tilde{U}_{b}$ that count the number of overflow and underflow events across the renewal intervals (recall Section IV). To this end, we define a random process that counts the number of renewals (i.e., events $\left\{C_{j}=0\right\}$ ) up to time $i$, namely:

$$
N(i)=\left|\left\{j \in\{1, \ldots, i\}: C_{j}=0\right\}\right|,
$$

where $|\cdot|$ represents the cardinality of its argument. It is also convenient to classify the renewal events depending on the value of the battery at the beginning of the renewal interval. We can then define

$$
N_{b}(i)=\mid\left\{j \in\{1, \ldots, i\}: C_{j}=0 \text { and } B_{j}=b\right\} \mid .
$$

The relationship between (41) and (42) is given as

$$
N(i)=\sum_{b=0}^{B_{\max }} N_{b}(i)
$$

Moreover, the initial time instant of the $i$ th interval corresponding to an initial battery state $b \in\left\{0, \ldots, B_{\max }\right\}$ can be written as

$$
S_{b, j}=\min \left\{i: N_{b}(i)=j\right\} .
$$

Using (41)-(44), we can now obtain the relationship (see also [28, pp. 239-240])

$$
\frac{\sum_{b=0}^{B_{\max }} \sum_{j=1}^{N_{b}(i)} \tilde{U}_{b, j}}{i} \leq \frac{\sum_{j=1}^{i} U_{j}}{i} \leq \frac{\sum_{b=0}^{B_{\max }} \sum_{j=1}^{N_{b}(i)+1} \tilde{U}_{b, j}}{i},
$$

$$
\begin{aligned}
& \underset{m \neq 0,1, B_{\max }}{\tilde{p}_{m, m-n}}=\sum_{i=d+1}^{k+1} p_{I}(i) \cdot \begin{cases}\left(1-p_{10}\right) p(0 ; i, q) & n=-1 \\
\left(1-p_{10}\right) q \sum_{l=n}^{i-1} p(l ; i-1, q)+p_{10} \sum_{l=n}^{i} p(l ; i, q) & n=m \\
\left(1-p_{10}\right)[q p(n ; i-1, q)+(1-q) p(n+1 ; i-1, q)] & \\
+p_{10} p(n ; i, q) & n=0,1, \ldots, m-1,\end{cases} \\
& \underset{\substack{m, m-n \\
m=0}}{\tilde{p}}=\sum_{i=d+1}^{k+1} p_{I}(i) \cdot \begin{cases}\left(1-p_{10}\right)(1-q) \sum_{l=n+1}^{i-1} p(l ; i-1, q) & n=-1 \\
\left(1-p_{10}\right) q \sum_{l=n}^{i-1} p(l ; i-1, q)+p_{10} \sum_{l=n}^{i} p(l ; i, q) & n=0,\end{cases} \\
& =\sum_{i=d+1}^{k+1} p_{I}(i) \cdot \begin{cases}\left(1-p_{10}\right)(1-q) & n=-1 \\
\left(1-p_{10}\right) q+p_{10} & n=0,\end{cases} \\
& \tilde{p}_{m, m-n}=\sum_{i=d+1}^{k+1} p_{I}(i) \cdot \begin{cases}\left(1-p_{10}\right) p(0 ; i, q) & n=-1 \\
\left(1-p_{10}\right)\left[q p(n ; i-1, q)+(1-q) \sum_{l=n+1}^{i-1} p(l ; i-1, q)\right] & \\
+p_{10} p(0 ; i, q) & n=0 \\
\left(1-p_{10}\right) q \sum_{l=n}^{i-1} p(l ; i-1, q)+p_{10} \sum_{l=n}^{i} p(l ; i, q) & n=1,\end{cases} \\
& \tilde{p}_{m=B_{\max }}=\sum_{i=d+1}^{k+1} p_{I}(i) \cdot\left\{\begin{aligned}
\left(1-p_{10}\right)[p(0 ; i, q)+q p(0 ; i-1, q)+(1-q) p(1 ; i-1, q)] & \\
\quad+p_{10} p(0 ; i, q) & n=0 \\
\left(1-p_{10}\right)[q p(n ; i-1, q)+(1-q) p(n+1 ; i-1, q)] & \\
\quad+p_{10} p(n ; i, q) & n=1,2, \ldots m-2 \\
\left(1-p_{10}\right)\left[q p(n ; i-1, q)+(1-q) \sum_{l=n+1}^{i-1} p(l ; i-1, q)\right] & \\
\quad+p_{10} p(n ; i, q) & n=m-1 \\
\left(1-p_{10}\right) q \sum_{l=n}^{i-1} p(n ; i-1, q)+p_{10} \sum_{l=n}^{i} p(n ; i, q) & n=m
\end{aligned}\right.
\end{aligned}
$$


where

$$
\tilde{U}_{b, j}=\sum_{k=S_{b, j-1}}^{S_{b, j}} U_{k}
$$

Averaging over all battery states $b$, we also have

$$
\mathrm{E}\left[\tilde{U}_{b}\right]=\sum_{i=d+1}^{k+1} p_{I}(i) \mathrm{E}\left[\tilde{U}_{b, i}\right]
$$

The left hand side of (45) can be separated as

$$
\frac{\sum_{b=0}^{B_{\max }} \sum_{j=1}^{N_{b}(i)} \tilde{U}_{b, j}}{i}=\frac{\sum_{b=0}^{B_{\max }} \sum_{j=1}^{N_{b}(i)} \tilde{U}_{b, j}}{N(i)} \frac{N(i)}{i} .
$$

Therefore, $t \rightarrow \infty$, since we have $N(t) \rightarrow \infty$, the strong law of renewal processes can be invoked on the second term on the right hand side of (48) to conclude that $N(i) / i \rightarrow 1 / \mathrm{E}[I]$ with probability one [28]. As for the first term, it can be written as

$$
\begin{aligned}
\frac{\sum_{b=0}^{B_{\max }} \sum_{j=1}^{N_{b}(i)} \tilde{U}_{b, j}}{N(i)} & =\sum_{b=0}^{B_{\max }} \frac{\sum_{j=1}^{N_{b}(i)} \tilde{U}_{b, j}}{\sum_{b^{\prime}=0}^{B_{\max }} N_{b^{\prime}}(i)} \\
& =\sum_{b=0}^{B_{\max }}\left(\frac{\sum_{j=1}^{N_{b}(i)} \tilde{U}_{b, j}}{N_{b}(i)} \frac{N_{b}(i)}{\sum_{b^{\prime}=0}^{B_{\max }} N_{b^{\prime}}(i)}\right)
\end{aligned}
$$

As a result, if $t \rightarrow \infty$, and hence $N_{b}(i) \rightarrow \infty$, by the strong law of large numbers, noting the fact that the random variables $\tilde{U}_{b, j}$ for every $b \in\left[0, B_{\max }\right]$ are i.i.d. across $j$, we have $\sum_{j=1}^{N_{b}(i)} \tilde{U}_{b, j} / N_{b}(i) \rightarrow \mathrm{E}\left[\tilde{U}_{b, j}\right]$ with probability one. Finally, by the law of large numbers for ergodic Markov chains (see, e.g., [24]), we have $N_{b}(i) / \sum_{b^{\prime}=0}^{B_{\max }} N_{b^{\prime}}(i) \rightarrow \tilde{\pi}_{b}$, where we recall that $\tilde{\pi}_{b}$ is the steady-state of the Markov chain $\tilde{B}_{i}$, which can be calculated from the transition probabilities detailed in Appendix A. From the discussion above, we conclude that the following limit holds with probability one:

$$
\lim _{i \rightarrow \infty} \frac{\sum_{b=0}^{B_{\max }} \sum_{j=1}^{N_{b}(i)} \tilde{U}_{b, j}}{i}=\frac{\sum_{b=0}^{B_{\max }}\left(\mathrm{E}\left[\tilde{U}_{b}\right] \cdot \tilde{\pi}_{b}\right)}{\mathrm{E}[I]}
$$

The same limit is obtained by applying the approach detailed above to the right-hand side of the inequality (45). This concludes the proof of (28) in Proposition 1. The overflow probability (27) is obtained following the same approach.

\section{REFERENCES}

[1] V. Chawla and S. H. Dong, "An overview of passive RFID," IEEE Commun. Mag., vol. 45, no. 9, pp. 11-17, Sep. 2007.

[2] F. Zhang et al., "Wireless energy transfer platform for medical sensors and implantable devices," in Proc. Annu. Int. Conf. IEEE EMBC, Minneapolis, MN, USA, Sep. 2009, pp. 1045-1048.
[3] A. Yakovlev, K. Sanghoek, and A. Poon, "Implantable biomedical devices: Wireless powering and communication," IEEE Commun. Mag., vol. 50, no. 4, pp. 152-159, Apr. 2012.

[4] P. V. Nikitin, S. Ramamurthy, R. Martinez, and K. V. S. Rao, "Passive tag-to-tag communication," in Proc. IEEE Int. Conf. RFID, Orlando, FL, USA, Apr. 2012, pp. 177-184

[5] W. C. Brown, "The history of power transmission by radio waves," IEEE Trans. Microw. Theory Tech., vol. MTT-32, no. 9, pp. 1230-1242, Sep. 1984.

[6] [Online]. Available: http://www.wirelesspowerconsortium.com/

[7] [Online]. Available: http://www.powercastco.com/

[8] L. R. Varshney, "Transporting information and energy simultaneously," in Proc. IEEE ISIT, Toronto, ON, Canada, Jul. 2008, pp. 1612-1616.

[9] P. Grover and A. Sahai, "Shannon meets Tesla: Wireless information and power transfer," in Proc. IEEE ISIT, Austin, TX, USA, Jun. 2010 , pp. 2363-2367.

[10] L. R. Varshney, "On energy/information cross-layer architectures," in Proc. IEEE ISIT, Cambridge, MA, USA, Jul. 2012, pp. 1356-1360.

[11] R. Zhang and C. K. Ho, "MIMO broadcasting for simultaneous wireless information and power transfer," in Proc. IEEE GLOBECOM, Houston, TX, USA, Dec. 2011, pp. 1-5.

[12] X. Zhou, R. Zhang, and C. K. Ho, "Wireless information and power transfer: Architecture design and rate-energy tradeoff," in Proc. IEEE GLOBECOM, Anaheim, CA, USA, Dec. 2012, pp. 3982-3987.

[13] Z. Xiang and M. Tao, "Robust beamforming for wireless information and power transmission," IEEE Wireless Commun. Lett., vol. 1, no. 4, pp. 372375, Aug. 2012.

[14] K. Huang and E. G. Larsson, Simultaneous Information and Power Transfer for Broadband Wireless Systems, 2012. [Online]. Available: http:// arxiv.org/abs/1211.6868

[15] J. Park and B. Clerckx, Joint Wireless Information and Energy Transfer in a Two-User MIMO Interference Channel, 2013. [Online]. Available: http://arxiv.org/abs/1303.1693v2

[16] K. Huang and V. K. N. Lau, Enabling Wireless Power Transfer in Cellular Networks: Architecture, Modeling, and Deployment, 2012. [Online]. Available: http://arxiv.org/abs/1207.5640

[17] Y. J. Zhang and K. B. Letaief, Optimal Scheduling and Power Allocation for Two-Hop Energy Harvesting Communication Systems, 2012. [Online]. Available: http://arxiv.org/abs/1212.5394

[18] K. Ishibashi, H. Ochiai, and V. Tarokh, "Energy harvesting cooperative communications," in Proc. IEEE Int. Symp. Pers., Indoor Mobile Radio Commun., Sydney, N.S.W., Australia, Sep. 2012, pp. 1819-1823.

[19] A. A. Nasir, X. Zhou, S. Durrani, and R. A. Kennedy, Relaying Protocols for Wireless Energy Harvesting and Information Processing, 2012. [Online]. Available: http://arxiv.org/abs/1212.5406

[20] P. Popovski, A. M. Fouladgar, and O. Simeone, "Interactive joint transfer of energy and information," IEEE Trans. Commun., vol. 61, no. 5, pp. 2086-2097, May 2013.

[21] A. M. Fouladgar and O. Simeone, "Information and energy flows in graphical networks with energy transfer and reuse," IEEE Wireless Commun. Lett., vol. 2, no. 4, pp. 371-374, Aug. 2013.

[22] L. Liu, R. Zhang, and K. C. Chua, "Wireless information transfer with opportunistic energy harvesting," IEEE Trans. Wireless Commun., vol. 12, no. 1, pp. 288-300, Jan. 2013.

[23] T. Cover and J. A. Thomas, Elements of Information Theory. Hoboken, NJ, USA: Wiley-Interscience, 2006.

[24] B. Marcus, R. Roth, and P. Siegel, Introduction to Coding for Constrained Systems. [Online]. Available: http://www.math.ubc.ca/ marcus/ Handbook/

[25] A. I. Barbero, E. Rosnes, G. Yang, and O. Ytrehus, "Constrained codes for passive RFID communication," in Proc. IEEE Inf. Theory Appl. Workshop, San Diego, CA, USA, Feb. 2011, pp. 1-9.

[26] A. Kailas and M. A. Ingram, "A novel routing metric for environmentallypowered sensors with hybrid energy storage systems," in Proc. Wireless VITAE Conf., Aalborg, Denmark, May 2009, pp. 1-6.

[27] D. O’Neill, M. Levorato, A. Goldsmith, and U. Mitra, "Residential demand response using reinforcement learning," in Proc. IEEE Int. Conf. SmartGridComm, Gaithersburg, MD, USA, Oct. 2010, pp. 409-414.

[28] R. G. Gallager, Discrete Stochastic Processes. Norwell, MA, USA: Kluwer, 1996.

[29] A. El Gamal and Y.-H. Kim, Network Information Theory. Cambridge, U.K.: Cambridge Univ. Press, 2011.

[30] S. Y. Kofman, "On the capacity of binary and Gaussian channels with runlength-limited inputs," IEEE Trans. Commun., vol. 38, no. 5, pp. 584-594, May 1990.

[31] E. Zehavi and J. K. Wolf, "On runlength codes," IEEE Trans. Inf. Theory, vol. 34, no. 1, pp. 45-54, Jan. 1988. 


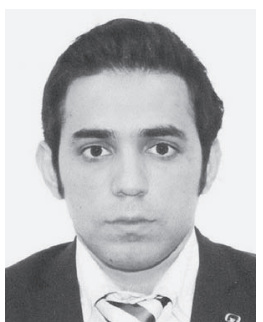

Ali Mohammad Fouladgar (S'14) received the B.Sc. and M.Sc. degrees in electrical engineering from Isfahan University of Technology, Isfahan, Iran, in 2006 and 2008, respectively. He is currently pursuing the Ph.D. degrees at the Center for Wireless Communications and Signal Processing Research (CWCSPR), New Jersey Institute of Technology (NJIT), Newark, NJ, USA. His current research interests concern the analysis and design of communication networks with emphasis on informationtheoretic and signal processing aspects.

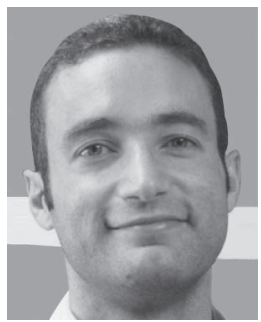

Osvaldo Simeone (M'02) received the M.Sc. degree (with honors) and the Ph.D. degree in information engineering from Politecnico di Milano, Milan, Italy, in 2001 and 2005, respectively. He is currently with the Center for Wireless Communications and Signal Processing Research (CWCSPR), New Jersey Institute of Technology (NJIT), Newark, NJ, USA, where he is an Associate Professor. His current research interests concern wireless communications, information theory and machine learning. Dr. Simeone is a co-recipient of Best Paper Awards of the IEEE SPAWC 2007 and IEEE WRECOM 2007. He currently serves as an Editor for IEEE Transactions on Information Theory and has previously served as an Editor for IEEE Transactions on Wireless Communications and IEEE Transactions on Communications.

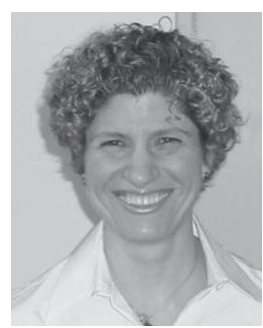

Elza Erkip (S'93-M'96-SM'05-F'11) received the B.S. degree in electrical and electronic engineering from Middle East Technical University, Ankara, Turkey, and the M.S. and Ph.D. degrees in electrical engineering from Stanford University, Stanford, CA USA. Currently, she is a Professor of Electrical and Computer Engineering at New York University Polytechnic School of Engineering, Brooklyn, NY, USA. Her research interests are in information theory, communication theory and wireless communications.

Dr. Erkip is a Fellow of the IEEE and a Member of the Science Academy Society of Turkey. She received the NSF CAREER award in 2001, the IEEE Communications Society Stephen O. Rice Paper Prize in 2004, the IEEE ICC Communication Theory Symposium Best Paper Award in 2007, and the IEEE Communications Society Award for Advances in Communication in 2013. She co-authored a paper that received the IEEE International Symposium on Information Theory Student Paper Award in 2007. Currently she is a Distinguished Lecturer and a Member of the Board of Governors of IEEE Information Theory Society.

Dr. Erkip is a Guest Editor of IEEE Journal on Selected Areas in Communications in 2014. She was an Associate Editor of IEEE Transactions on Information Theory from 2009-2011, an Associate Editor of IEEE Transactions on Communications from 2006-2008, a Publications Editor of IEEE Transactions on Information Theory from 2006-2008 and a Guest Editor of IEEE Signal Processing Magazine in 2007. She was a General Chair of IEEE International Symposium of Information Theory in 2013, a Technical Program Chair of International Symposium on Modeling and Optimization in Mobile, Ad Hoc, and Wireless Networks (WiOpt) in 2011, a Technical Program Chair of IEEE GLOBECOM Communication Theory Symposium in 2009, the Publications Chair of IEEE Information Theory Workshop, Taormina in 2009, the Technical Area Chair for the MIMO Communications and Signal Processing track of Asilomar Conference on Signals, Systems, and Computers in 2007, and a Technical Program Chair of IEEE Communication Theory Workshop in 2006. 